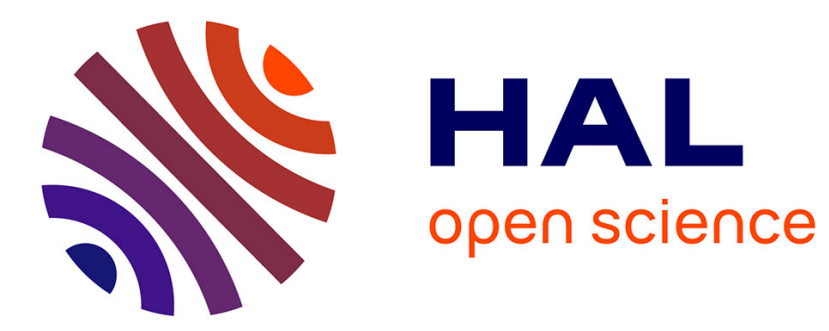

\title{
Ecologie de l'Alisier torminal Sorbus torminalis (L.) Crantz
}

Nicolas Drapier

\section{To cite this version:}

Nicolas Drapier. Ecologie de l'Alisier torminal Sorbus torminalis (L.) Crantz. Revue forestière française, 1993, 45 (3), pp.229-242. 10.4267/2042/26418. hal-03443994

\section{HAL Id: hal-03443994 \\ https://hal.science/hal-03443994}

Submitted on 23 Nov 2021

HAL is a multi-disciplinary open access archive for the deposit and dissemination of scientific research documents, whether they are published or not. The documents may come from teaching and research institutions in France or abroad, or from public or private research centers.
L'archive ouverte pluridisciplinaire HAL, est destinée au dépôt et à la diffusion de documents scientifiques de niveau recherche, publiés ou non, émanant des établissements d'enseignement et de recherche français ou étrangers, des laboratoires publics ou privés. 


\title{
ÉCOLOGIE DE L'ALISIER TORMINAL SORBUS TORMINALIS (L.) CRANTZ
}

\author{
N. DRAPIER
}

\section{CONTEXTE DE L'ÉTUDE}

L'intérêt des forestiers et scientifiques s'est porté sur l'Alisier torminal depuis quelques années que la hausse vertigineuse des cours de son bois en a fait l'essence forestière indigène la plus chère de France. C'est cette préoccupation toute prosaïque qui a permis le lancement d'un programme de recherche destiné à mieux connaître et valoriser l'Alisier.

Connaissance essentielle à l'élaboration d'une sylviculture, l'écologie de l'Alisier était considérée comme méconnue: on savait que l'espèce avait une grande amplitude écologique, et guère plus.

Au terme de l'étude que nous avons eu à en faire, ceci appelle déjà quelques remarques.

En Allemagne (où l'intérêt pour cette essence s'est manifesté dès le début des années 70 ) et en Suisse, l'Alisier torminal a déjà fait l'objet de travaux importants (Kausch-Blecken von Schmeling et Meyer, 1980 et 1981 ; Pleines, 1981). Des conditions de milieu largement différentes font que leurs résultats ne sont que très incomplètement transposables à la France. Cependant, dans l'hypothèse où il n'aurait pas été possible de lancer un programme de recherche en France (cela tient à peu de choses), on peut se demander si l'on aurait au moins cherché à appliquer aux forèts de notre pays les résultats de ces études étrangères.

De même, on n'insistera jamais trop sur la richesse d'enseignements de certains classiques de la littérature forestière (Baudrillart, Mathieu, Lorentz et Parade, Guinier et al. ...). Dans l'attente de résultats des recherches nouvellement lancées, ou éventuellement à défaut de celles-ci, il s'y trouvait déjà assez d'éléments pour mettre en place une sylviculture élémentaire de l'Alisier torminal. C'est-à-dire au moins de quoi éviter, quant au choix de conditions de milieu et de conduite des individus et peuplements, le genre d'erreurs qu'on a déjà vu commettre pour d'autres essences.

Cependant, les classiques colportent aussi des erreurs qui semblent parfois avoir la vie plus dure que ce qu'ils contiennent de judicieux, et qui suffiraient à justifier la réalisation de nouvelles études. II faut aussi s'y méfier de connaissances régulièrement citées depuis sans avoir vraiment été actualisées: ainsi pour l'énumération des usages traditionnels de l'alisier, maintenant désuets ou mineurs (mécanique, instruments de musique...), tandis que son excellence comme bois d'ébénisterie est relativement méconnue, et ce marché encore peu développé.

Nombre de publications plus récentes (ouvrages généraux de dendrologie ou de sylviculture, typologies forestières...) comportent aussi des erreurs ou inexactitudes sur l'écologie et la sylviculture de l'Alisier, pour avoir généralisé abusivement les données propres à une région d'étude limitée, ou bien ne pas avoir pris en compte certains aspects synécologiques ou 
historiques (voir plus loin). C'est aussi le cas de quelques publications plus spécialisées auxquelles le récent regain d'intérêt pour l'Alisier torminal a déjà fourni matière, et quỉ ont pâti d'un très compréhensible manque d'informations générales (comme premiers travaux importants sur l'Alisier torminal en France, voir les études de Mauranges, 1981 et de Nicloux, 1986). II sera question plus loin de ce problème de l'exploitabilité de la documentation préexistante.

Précisons enfin que, partant de peu de choses et s'intéressant à tout le territoire français, notre étude de l'écologie de l'Alisier torminal a eu pour objectif une connaissance générale, qui puisse ouvrir la voie à des travaux plus précis (portant en particulier sur des territoires restreints) et déjà être utile aux forestiers. C'est pourquoi nous nous sommes imposé beaucoup de circonspection, et avons voulu que cet article soit essentiellement pratique.

\section{REMARQUES MÉTHODOLOGIQUES}

L'étude de l'écologie d'une essence telle que l'Alisier torminal se heurte à des particularités faisant que l'exploitation de la bibliographie ne peut être fructueuse:

- Sensibilité à la concurrence: donnée essentielle (voir plus loin), qui entraîne un comportement très variable de l'espèce selon le type de végétation et le traitement sylvicole - avant même que les autres facteurs du milieu n'aient à entrer en considération. Toute indication sur la rareté ou la plus ou moins bonne venue de l'Alisier torminal, sans plus de précisions sur les conditions de milieu et de sylviculture (présente et antérieure) s'en trouve donc complètement sujette à caution.

- Rareté (souvent directement liée au précédent facteur) : il aura souvent été constaté, au cours de notre étude, que l'Alisier existe (et même sous la forme de sujets remarquables) dans des territoires ou milieux où il était considéré comme absent.

- Dissémination : elle impose de disposer de localisations très précises pour les prospections - ce que la littérature ne fournit que rarement.

II est à noter que les catalogues de stations sont plus utiles que les autres sources bibliographiques, parce qu'ils constituent des ètudes générales et synthétiques sur la végétation forestière de régions entières. Ce sont des sources documentaires sans équivalent (notamment sur l'histoire sylvicole et la pédologie), grâce à une démarche phytoécologique qu'on ne trouve en particulier guère dans la littérature phytosociologique classique.

Cependant, dans la perspective de l'étude d'une essence secondaire et rare, les catalogues de stations présentent eux aussi des lacunes importantes :

- par rapport à un travail de prospection spécifique, risque important d'omission de situations stationnelles intéressantes;

- de ce fait, indications souvent erronées (quand il y en a) sur les potentialités (et la répartition) de l'essence en question.

En conséquence, l'emploi d'un catalogue peut surtout être intéressant dans la perspective de l'étude d'une essence à l'échelle de la seule région concernée. L'étude peut alors être faite en fonction des types stationnels ainsi prédéfinis (et même bénéficier des prospections faites à cette occasion pour disposer d'un échantillonnage aussi proche que possible de l'exhaustivité). Toutefois, l'efficacité de la démarche consistant à se référer aux types stationnels est très variable, car elle dépend en particulier de la façon dont certains facteurs écologiques importants ont pu ou non être pris en compte dans la réalisation du catalogue; ainsi pour l'hydromorphie, dont l'ètude rigoureuse nécessite des moyens assez lourds qui ne sont habituellement pas mis en œuvre dans ce cadre; dès lors, l'étude des relations station-production d'une essence dans de telles conditions de milieu a tendance à devoir être faite indépendamment des catalogues (voir l'étude de Girard). 
Ces divers problèmes documentaires ont confirmé, au commencement de notre étude:

- la nécessité de prospections: l'étude bibliographique, même très générale, de l'écologie d'une essence, ne peut être conduite qu'avec toutes les réserves que nous avons faites sur la validité de ce type de données - ne serait-ce qu'en ce qui concerne la distribution géographique de l'espèce, et beaucoup plus encore quant à ses potentialités. Et encore, ne peut-on pas en attendre beaucoup de résultats.

- le besoin de renseignements plus précis que ceux fournis par la bibliographie pour l'établissement du plan d'échantillonnage.

En conséquence, nous avons lancé une enquête auprès d'organismes concernés (ONF, DDAF, SERFOB, CRPF, laboratoires de recherche), en accordant un soin particulier à la conception d'un questionnaire simple (et donc pas rebutant); il fut également précisé que des réponses très brèves nous suffiraient (y compris négatives) et que cette recherche concernait l'Alisier torminal sous toutes ses formes (pas uniquement les beaux sujets, dont il s'avéra d'ailleurs que la conception était très variable selon les régions et les personnes).

Pour plus de 330 envois réalisés, près de 150 réponses ont été obtenues, taux de retour élevé qui semble confirmer la pertinence de nos précautions. Les données recueillies ont été suffisamment abondantes, précises et bien réparties, pour permettre l'élaboration de tout le plan de prospection. Le succès de cette enquête, dont nous sommes redevables à tous les forestiers qui y ont répondu, aura donc été essentiel pour la suite de l'étude (conduite, faut-il préciser, en étroite collaboration avec celle de E. Sevrin sur la qualité du bois d'alisier).

\section{ASPECTS SYNÉCOLOGIQUES DE LA BIOLOGIE DE L'ALISIER TORMINAL}

\section{Comportement dynamique}

L'Alisier torminal a un tempérament dynamique d'espèce post-pionnière (1). 11 est intéressant de comparer ses caractéristiques architecturales avec celles de pionnières ${ }^{(2)}$ et de dryades ${ }^{(3)}$.

L'Alisier blanc, le Sorbier des Oiseleurs, l'Alisier de Fontainebleau (voir l'article "Écologie et intérêt sylvicole de divers Sorbus en France" pour les détails, p. 345) ont un comportement nettement plus pionnier que l'Alisier torminal. Par rapport à celui-ci, et en cohérence avec les exigences de leur comportement dynamique, ces trois espèces ont une bien meilleure capacité à rejeter de souche, se ramifier, émettre des gourmands, voire se marcotter - donc à " repartir " après toutes sortes de traumatismes (sécheresse, bris de neige, abroutissement, mouvement du substrat...). Elles sont en outre très héliophiles, et supportent mal à pas du tout la concurrence.

À l'opposé, les dryades (Hêtre, Sapin...) se caractérisent en général par leur aptitude à produire un tronc unique s'élevant à travers un couvert préexistant. En attendant ce moment, elles supportent l'ombre et la concurrence, en particulier lors de la phase critique de régénération.

On peut rapprocher de cette catégorie certaines espèces post-pionnières que nous qualifierons de "persistantes": le Merisier, l'Érable sycomore (surtout) sont capables de supporter un couvert assez fermé, et de croître jusqu'à s'affranchir de ces contraintes.

(1) Post-pionnière: qualifie une essence intervenant dans les successions vègètales après les essences pionnières, et normalement avant les dryades.

(2) Pionnière : se dit d'une essence apte à coloniser des milieux ouverts, perturbès, instables, et participant donc aux stades initiaux d'une sylvigènèse.

(3) Dryade: essence caractéristique des phases de maturation d'une sylvigénèse. 


\section{N. DRAPIER}

En comparaison, nous considérerons l'Alisier torminal comme une post-pionnière "primitive ": il supporte mal la concurrence, ne peut guère que végéter sous couvert, où il disparaît rapidement si cette contrainte se maintient. II est aussi nettement héliophile, mais n'atteint pas l'aptitude au nomadisme ${ }^{(4)}$ des trois autres Sorbus cités plus haut. Et même s'il est "plus forestier" que ceux-ci, ce n'est pas au point de mériter le qualificatif d'essence de "demi-ombre " qu'on lui donne souvent; ce terme est à réserver, par exemple, aux Grands Érables, au Merisier, ou au Charme.

\section{Réaction de l'Alisier torminal à la concurrence et aux structures de peuplements}

Nous plaçons et développons ce sujet avant celui du comportement autécologique de l'Alisier, parce que c'est bien la structure des peuplements qui conditionne en premier lieu l'état sous lequel s'y présente actuellement cette essence. Primant sur sa grande rusticité (voir $\S$ Autécologie de l'Alisier torminal, p. 233), la sensibilité à la concurrence de l'Alisier torminal a en effet pour conséquences:

- Sa grande rareté, voire son éviction (cas fréquent) :

- de milieux (très) fertiles où le dynamisme d'autres essences lui laisse très peu de chances ;

- des futaies régulières en général - mais uniquement à cause de l'absence de soins dont l'Alisier a jusqu'à maintenant été victime dans ce type de peuplements.

- La présence habituelle de l'Alisier, et son abondance:

- dans des peuplements plus ou moins bas et ouverts, c'est-à-dire souvent dégradés à cause de conditions stationnelles défavorables et de gestion négligée ;

- dans les taillis-sous-futaies, dont la structure hétérogène est plus favorable que celle des futaies régulières, et où l'on a d'ailleurs plus volontiers recruté les fruitiers en baliveau par le passé ; mais il est à noter que cette tradition n'a pas touché également toutes les régions de France, et qu'elle s'est plus ou moins perdue dans certaines avec le développement de peuplements réguliers et pauvres spécifiquement.

- Corollairement, les impressions erronées que :

- l'Alisier torminal est un petit arbre inutilisable en futaie régulière; il y atteint (évidemment exceptionnellement, en l'état actuel des choses) plus de $30 \mathrm{~m}$;

- ne lui conviennent que certains milieux difficiles; ceci est également inexact, comme on le verra plus loin.

Les figures des pages 234 à 237 illustrent des situations écologiques - au sens le plus large, incorporant jusqu'aux facteurs anthropiques - dans lesquelles nous avons observé l'Alisier torminal. On y a joint l'interprétation que nous faisons de leurs conséquences sur la situation de l'Alisier et sur les possibilités de sa mise en valeur dans ces divers milieux (voir l'article "Recherche d'éléments de sylviculture pour l'Alisier torminal", p. 321).

Cette approche implique la prise en compte de l'action directe ou indirecte de plusieurs facteurs :

- composition dendrologique du peuplement ;

- effet des conditions stationnelles sur le développement de l'Alisier et des autres essences;

- effets de la sylviculture, passée ou à venir: traitement, interventions (dépressages, élagages...), actions particulières. Nous avons figuré des situations inédites, correspondant à des possibilités hypothétiques de développement de la sylviculture de l'Alisier.

Les situations présentées sont classées en premier lieu par type de traitement sylvicole (taillissous-futaie, futaie, taillis simple), ce facteur étant apparu comme le plus important. Viennent

(4) Nomade: se dit d'une essence post-pionnière ou dryade pouvant jouer un rôle de pionnière dans certaines conditions. 
ensuite l'influence d'interventions particulières (traditionnelles en général) et celles de facteurs stationnels et de la composition dendrologique. Ces indications peuvent être considérées comme des éléments d'aide au diagnostic pour la gestion de peuplements.

\section{Réaction à d'autres facteurs biotiques}

La composition dendrologique n'est pas le seul facteur écologique biotique dont dépend la situation de l'Alisier torminal dans les peuplements forestiers ou autres formations végétales. Ainsi :

- Les fruits de l'Alisier torminal sont appréciés des oiseaux qui en disséminent les semences. La relation avec le comportement dynamique de l'espèce est évidente, tant par la capacité de conquête ainsi conférée à l'Alisier, que par la façon dont cela le destine à surtout s'implanter dans des milieux bien fréquentés par les oiseaux frugivores (lisières et trouées par exemple).

- L'abroutissement de l'Alisier par les cervidés est très fréquent. La prédation peut aussi être le fait de rongeurs, en forêt comme dans les fruticées (ce qui semble limiter la place que l'Alisier torminal peut tenir dans ces milieux très ouverts).

- L'Alisier a divers parasites animaux (pucerons, acariens) et il est sensible à des maladies cryptogamiques (voir l'article de L. Lanier, p. 343). Ces dégâts peuvent être importants, et l'on a vu, à l'occasion du dépérissement observé ces dernières années, que les alisiers ainsi affaiblis sont sensibles au pourridié. II est possible que cette sensibilité à ces risques phytosanitaires (plus grande que chez beaucoup d'autres essences) ait aussi une part de responsabilité dans le caractère toujours disséminé de l'Alisier torminal dans les peuplements.

\section{AUTÉCOLOGIE DE L’ALISIER TORMINAL}

\section{Remarque préliminaire}

L'autécologie est l'ètude des relations de l'individu (ou de l'espèce) avec les facteurs du milieu; cette notion est complétée par celle de synécologie, qui s'applique aux communautés d'êtres vivants (déterminisme et organisation).

L'usage a souvent fait prendre la notion d'autécologie d'une espèce végétale comme l'étude des relations de celle-ci avec les seuls facteurs écologiques abiotiques. Or, il a été montré précédemment à quel point cela est réducteur et source d'erreurs d'interprétation. Nous nous proposons donc, soit de conserver à l'autécologie ce sens restreint, à condition de parler de même de la synécologie d'une espèce (étude des relations de l'espèce avec son environnement biotique, donc de sa place dans une communauté); soit, pour le développement suivant qui ne traite que des relations de l'Alisier avec les facteurs abiotiques, de préciser qu'il s'agit de l'autécologie abiotique de l'espèce.

\section{Influence sur l'Alisier torminal de facteurs écologiques abiotiques}

\section{- Facteurs pédoclimatiques}

\section{Sécheresse}

La tolérance de l'Alisier torminal (et d'espèces voisines) à la sécheresse est meilleure que chez la plupart des feuillus indigènes des régions tempérées de France. Cependant, il n'est franchement ni xérophile, ni thermophile, comme l'atteste sa répartition gèographique; ce ne sont que les effets différenciés de la concurrence qui peuvent en donner l'impression. 
TAILLIS-SOUS-FUTAIE

Situation la plus fréquente en l'état actuel des choses, du fait :

- des surfaces encore importantes couvertes par ce type de peuplements,

- de l'adéquation souvent constatée entre leur traitement (nature et fréquence des interventions) et certaines exigences autécologiques (photiques surtout) de l'Alisier torminal.

\section{CAS OỦ DES INTERVENTIONS PASSÉES ONT PROFITÉ À L'ALISIER TORMINAL}

- Cas de stations moyennement fertiles à fertiles (ex. : plateaux calcaires et plaines marneuses du Nord-Est, certains matériaux de décarbonatation du Bassin Parisien...)

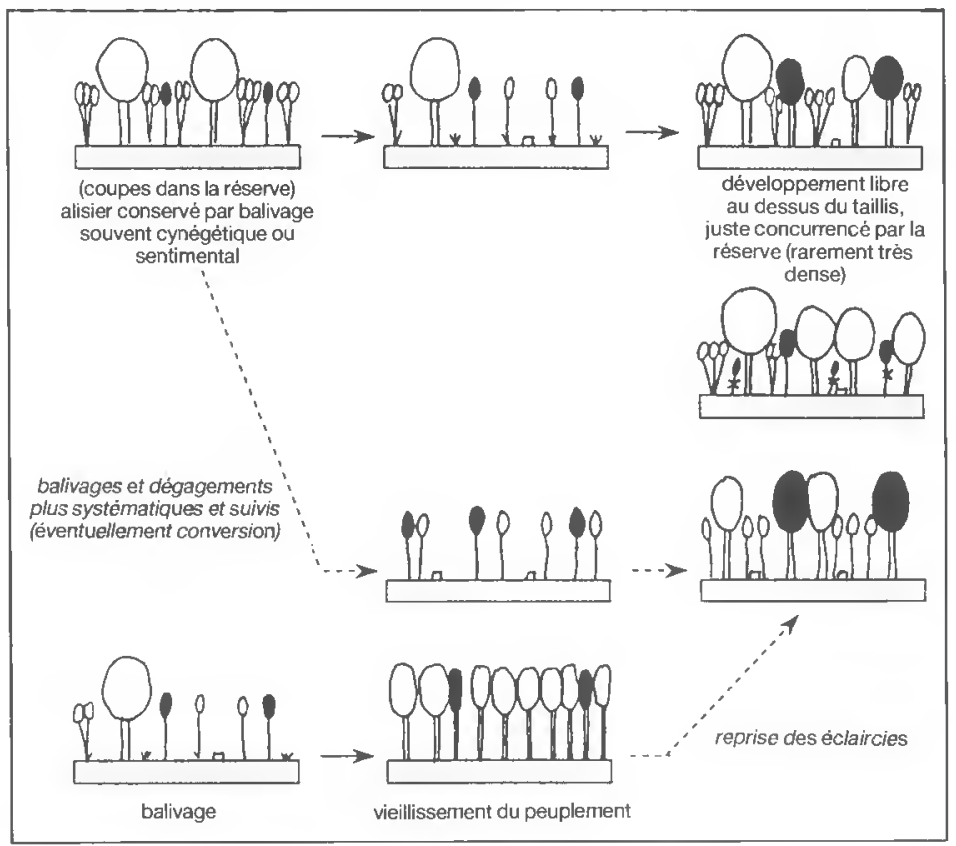

alisiers assez nombreux et bien conformés, le plus souvent codominants

- Cas de stations à fortes contraintes: hydromorphie, sécheresse, oligotrophie (ex. : sols superficiels des plateaux calcaires de Bourgogne ; sols plus ou moins acides et hydromorphes du Centre, Sud de la France en général...)

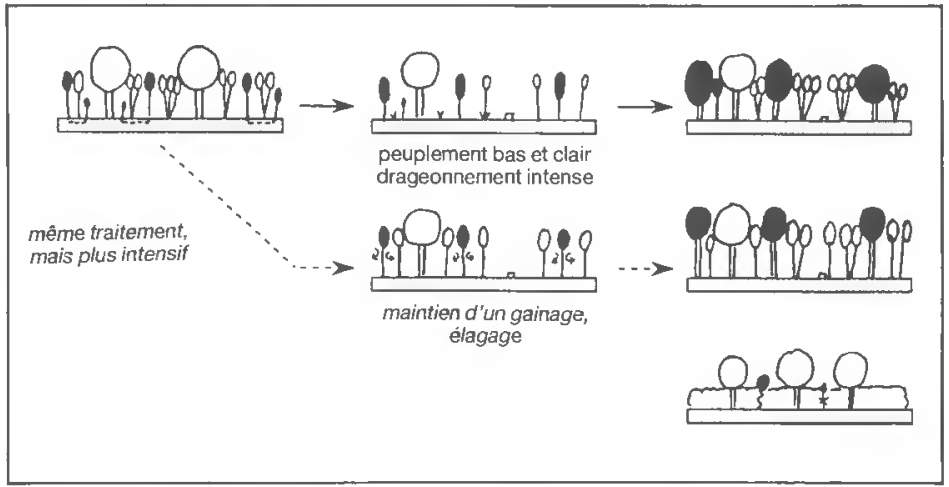

alisiers fort nombreux mais bas branchus

arbres moins courts, de plus grande valeur (même en restant en TSF)

variante : forte concurrence (buis p. ex.) (conditions stationnelles aggravantes) potentialités faibles, et 
- Cas de stations très fertiles (ex. : bas de pentes et fonds de vallons, sols sur lœess récent...)

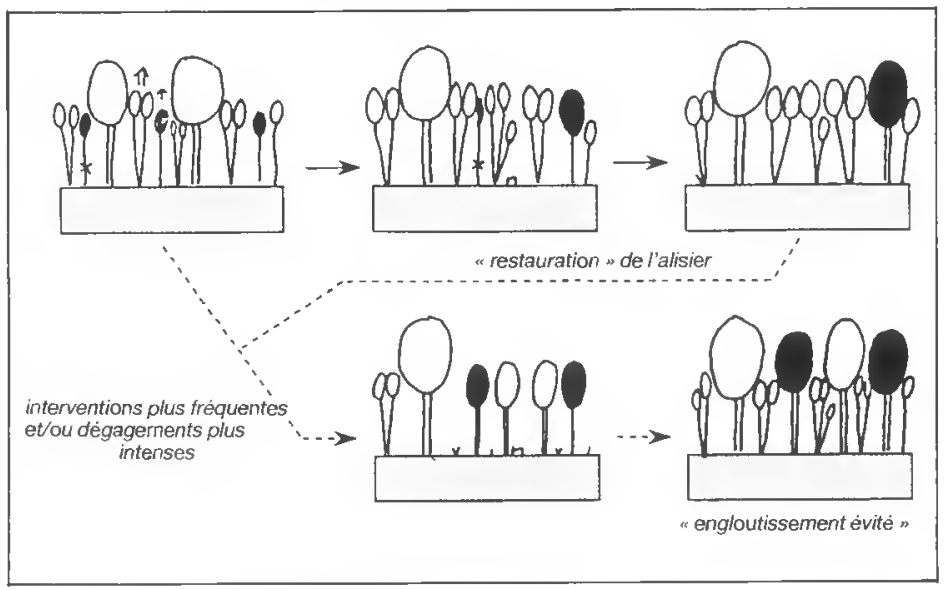
concurrence extrême, même si l'alisier a èté balivè : sujets parfois aussi beaux que rares
mais souvent tout à fait absents (potentialités masquèes)

- Cas particuliers de torte concurrence du taillis

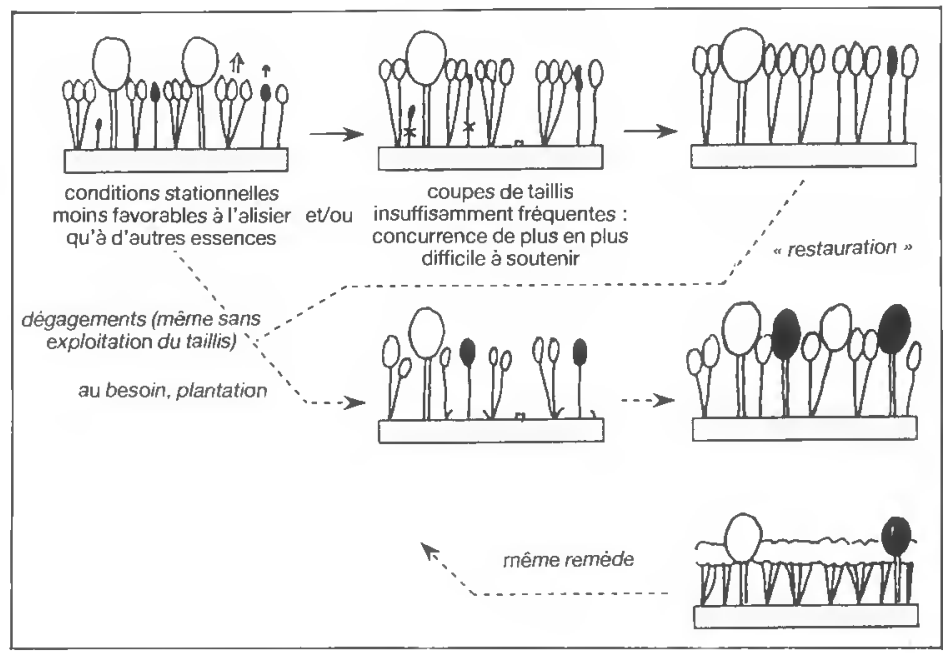

très beaux alisiers, à la mesure des potentialités de la station (sans qu'il soit nècessaire d'abandonner le TSF)

taillis excessivement élevé, alisiers rares et souvent grêles (potentialites masquées)

(beaux alisiers)

CAS DE RÉGIONS SANS TRADITION DE CONSERVATION DE FEUILLUS TELS QUE L'ALISIER TORMINAL

Cette contrainte y prime sur l'influence des caractères stationnels.

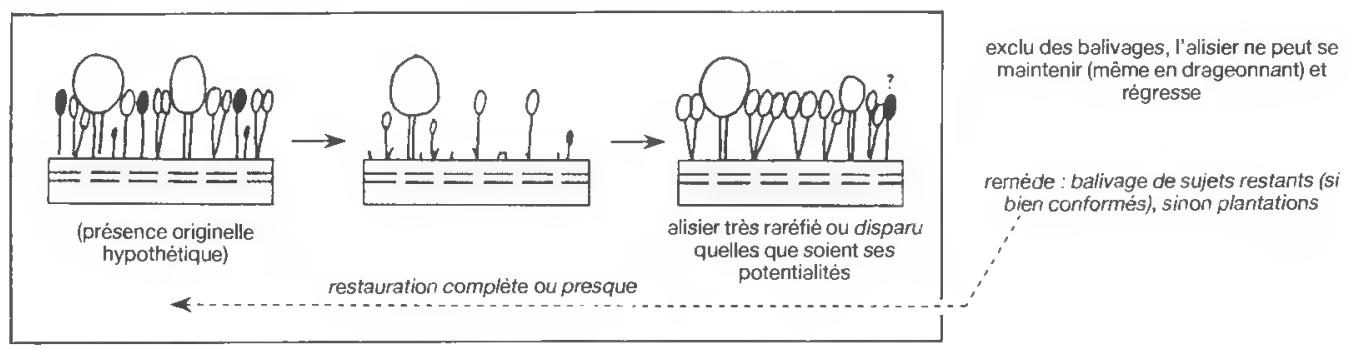
taillis pas particulièrement élevè, mais très dense et sombre (châtaignier, tilleul, charme dans certains cas). Alisiers encore rares.




\section{N. DRAPIER}

\section{FUTAIE}

En futaie régulière, l'Alisier torminal est rare actuellement, car le traitement est trop défavorable à cette espèce en l'absence d'égards pour elle (ce qui a toujours ou presque été le cas).

Quant à la futaie irrégulière, les exemples sont encore plus rares. Des cas de TSF convertis s'y apparentent, mais avec une distribution incomplète des classes d’âge. De la même façon, certains des développements envisageables à partir de situations existantes (en taillis-sous-futaie, futaie régulière, ou taillis simple) peuvent aboutir à une structure de futaie irrègulière plus ou moins complète.

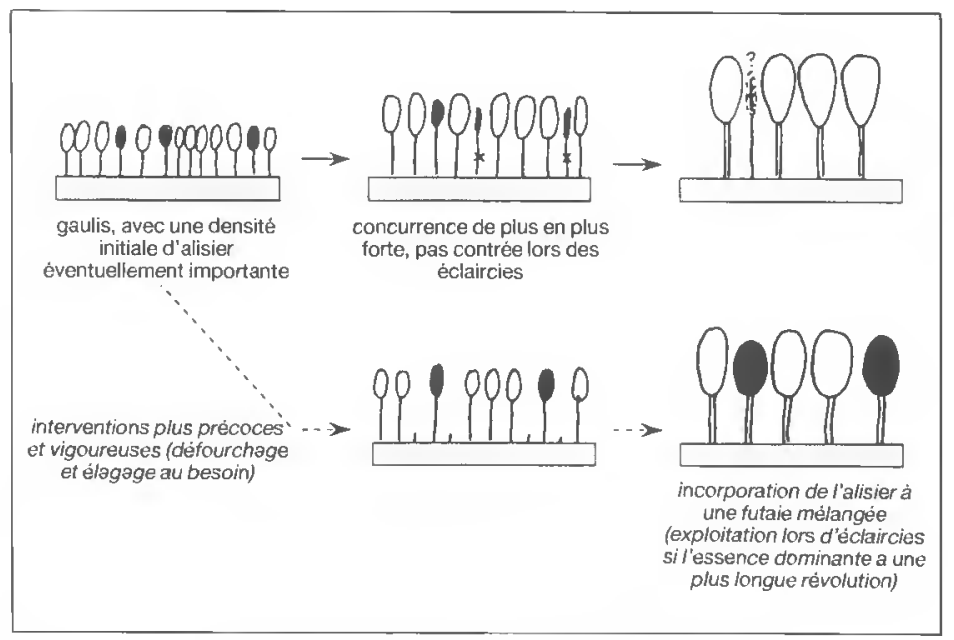

\begin{abstract}
cas typique : alisiers conserves exceptionneliement au fil des èclaircies successives, voire ètouffés entre deux interventions : individus très rares et grêles
\end{abstract}

alisiers de futaie, élancés et à bille longue

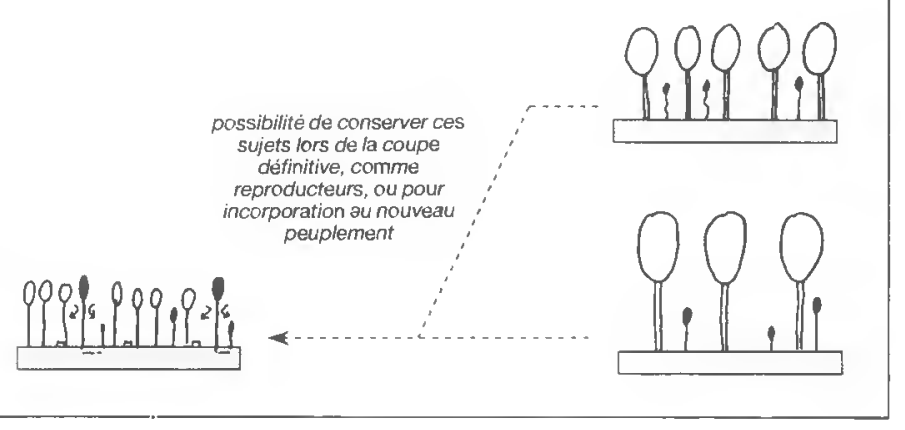

cas particulier : maintien en sousétage d'alisiers même très dominés, au moins à des fins cynégétiques (Centre)

\section{RAPPEL : DIFFÉRENTS STATUTS D'UN ARBRE AU SEIN D'UN PEUPLEMENT}

Situation dominee : arbre recouvert par les houppiers voisins. Fatal à plus ou moins courte échéance à l'alisier torminal s'il n'est pas dègagè.

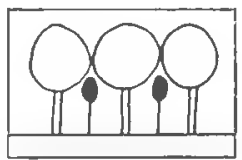

Situation codominante: l'arbre appartient à la strate supérieure mais peut être dépassé (et comprimé latéralement) par d'autres. Statut très fréquent pour l'alisier torminal, arbre de deuxieme grandeur (rarement plus de $30 \mathrm{~m}$ ), mais bien supporté par lui.

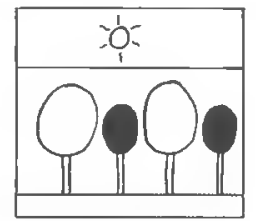

Situation prédominante : l'arbre égale les plus grands de la strate supérieure. Rare pour l'alisier torminal, presque toujours environné de plus grands sujets d'autres essences.

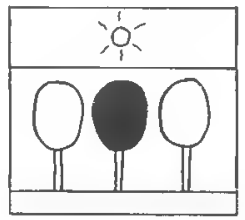




\section{TAILLIS SIMPLE}

Situation peu fréquente pour l'Alisier torminal. Son état dépend alors surtout des autres essences présentes, et des potentialités stationnelles.

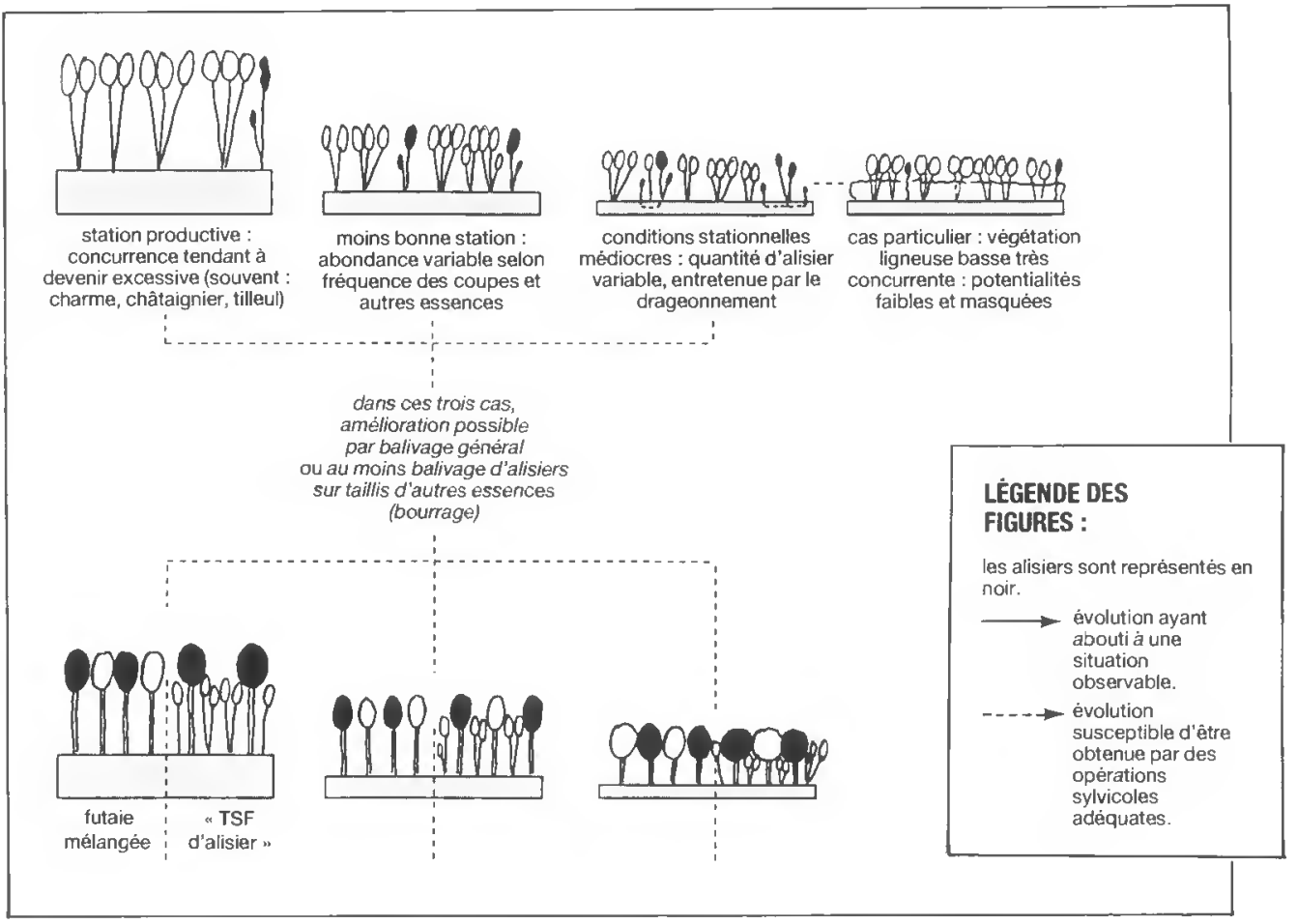

En outre, l'Alisier supporte nettement mieux la sécheresse quand ne s'y ajoute pas la contrainte de l'oligotrophie (donc sur calcaire mieux que sur substrat acide, voir $\S$ Nutrition minérale, p. 239).

Dans le bois de l'Alisier torminal, Lachaud et Mansouri (voir l'article p. 279) ont mis en évidence une abondance de trachéides, et des ornementations spiralées de la paroi intérieure des vaisseaux, qui sont une adaptation de la conduction de la sève brute en conditions de stress hydrique.

Hydromorphie

L'Alisier torminal a une bonne tolérance aux conditions d'engorgement temporaire, même intenses.

Contrastes pédoclimatiques

La tolérance de l'Alisier vis-à-vis de cette contrainte est particulièrement développée, dans l'absolu, et surtout par rapport aux autres essences habituellement présentes. Significativement, dans l'Est de la France, où l'Alisier torminal est presque absent des milieux à sols acides, c'est principalement lorsque ceux-ci présentent un régime hydrique contrasté qu'on retrouve l'Alisier (sur pélosols-pseudogleys, surtout, voir pp. 240-241).

Ce type de situation pédoclimatique appelle la même remarque que plus haut à propos de l'influence de la nutrition minérale. 


\section{N. DRAPIER}

- Froid

La répartition de l'Alisier torminal en France et en Europe atteste qu'il supporte de basses températures. En particulier, sous le climat du Nord-Est, ses jeunes feuilles et pousses résistent bien aux gelées printanières (au printemps 1991, par exemple, il les a beaucoup mieux supportées que les Chênes). En pépinière, on a vu des bourgeons non écailleux supporter des froids de $-15^{\circ} \mathrm{C}$.

Cependant, le froid est responsable, au moins partiellement, de l'absence de l'Alisier en montagne et de sa disparition dans le Nord de la France. Mais dans les deux cas, c'est moins le fait de l'existence d'un seuil physiologique que d'une diminution du dynamisme de l'espèce, au point cette fois que la concurrence la fasse disparaître (comme l'indique le fait qu'il y ait quelques très beaux arbres en Picardie, pourtant à la limite de l'aire). Dans ces conditions, la limitation tiendrait plus à un défaut de chaleur durant la saison de végétation qu'aux valeurs hivernales extrêmes (mais l'hypothèse, avancée par Roisin, d'un défaut de maturation des fruits sous ce climat, nous paraît insuffisante et de peu de poids face aux autres facteurs dynamiques).

\section{- Lumière}

Le plein découvert n'est pas une contrainte pour l'Alisier torminal (sauf peut-être dans les régions méridionales au climat chaud et $\mathrm{sec}$ ) ; c'est dans ces conditions que l'on a observé les meilleures croissances en diamètre.

En revanche, l'ombre, telle qu'en situation surcimée (voir p. 230), est assez rapidement fatale à l'Alisier. Une petite trouée dans la canopée, on l'a vu, lui sutfit généralement pour se maintenir.

- Synthèse: réactions aux tendances macroclimatiques

Montagnarde

La tolérance de l'Alisier y est médiocre, sa présence spontanée étant à peu près limitée à l'étage montagnard inférieur (avec une altitude atteinte plus élevée dans le Sud du pays). Cela n'exclut cependant pas que l'Alisier ait des potentialités intéressantes à des altitudes supérieures à cette limite, surtout à des expositions tavorables (voir § Froid, ci-dessus).

Continentale

C'est dans le Nord-Est de la France que l'Alisier torminal a pour le moment donné les résultats les plus intéressants, et les éventuelles contraintes climatiques liées à l'influence continentale sont sans préjudice pour lui. Deux exceptions sont à noter:

- quand, en plaine, une situation de fond de vallon froid reproduit des conditions climatiques montagnardes;

- et quand la contrainte macroclimatique se trouve combinée à une forte déficience de la nutrition minérale (voir p. 240).

Atlantique

Le climat atlantique français convient tout à fait à l'Alisier torminal, à quelques réserves près (mais pas rédhibitoires):

- dans le Nord du pays (puis en Belgique et au-delà) où la limite d'aire actuelle (on ne peut dire naturelle) de l'Alisier se détache des côtes en direction de contrées à influence continentale (étés plus chauds) (voir $\S$ Froid, ci-dessus);

- dans certaines régions du Centre, où la combinaison d'un macroclimat doux et relativement sec à des contraintes pédoclimatiques a un effet négatif sur les potentialités de l'Alisier (tout en étant souvent favorable à sa présence abondante dans des formations peu denses);

- en Bretagne, où l'habituelle combinaison du climat uniforme et frais et de sols oligotrophes fait que l'Alisier est très rare sur de vastes superficies (mais là encore, ceci n'exclut pas son introduction, surtout à la faveur de conditions stationnelles ponctuellement meilleures). 


\section{Méditerranéenne}

L'Alisier torminal se ressent apparemment beaucoup plus de la contrainte xérique que thermique. Il est présent dans le Midi, jusque dans des milieux très secs de l'Estérel, ce qui est remarquable pour une essence ayant son optimum de productivité dans la moitié Nord du pays ; de plus, il y est d'apparence saine, même si ce n'est le plus souvent que sous une forme arbustive [on peut comparer cela à des cas où la conjonction de contraintes climatiques et édaphiques (sols lessivés très dégradés) produit des alisiers rampants!].

Dans le Sud de la France, à la faveur d'ubacs, de bas de pente ou de fonds de vallées, l'Alisier devrait avoir de bonnes potentialités jusque dans des régions ou milieux dont il est pour l'instant complètement absent.

Face à ce type de limitation climatique, on aura également intérêt à envisager l'emploi de l'Alisier blanc (souvent présent) ou du Cormier (plus rare mais plus intéressant) (voir l'article "Écologie et intérêt sylvicole de divers Sorbus en France », p. 345).

\section{- Facteurs de la nutrition minérale}

L'Alisier est indifférent aux pH élevés, à la présence de calcium échangeable dans le sol, et même de calcaire actif.

L'étude de la tolèrance de l'Alisier à l'oligotrophie mérite d'être approfondie. Ses besoins nutritifs sont importants (d'après Barnola et Louni et les résultats d'une expérience de semis que nous avons faite), mais souvent compensés par une meilleure tolérance que d'autres essences à des contraintes édaphiques associèes (hydromorphie, contrastes hydriques). En outre, l'Alisier dispose d'importantes capacités d'extraction des nutriments pour satisfaire ses besoins (Barnola et Louni), qui expliqueraient que ses potentialités restent bonnes tant que les conditions d'oligotrophie ne sont pas excessives ou qu'il peut, vraisemblablement, "profiter " d'autres essences présentes grâce au cycle biogéochimique. Quant aux pH bas, c'est un facteur qui n'est apparemment pas contraignant en soi, mais indissociable de l'oligotrophie (il ne semble pas non plus qu'il y ait de sensibilité de l'Alisier à la toxicité de certains ions métalliques dans ces conditions).

\section{Synthèse : comportement de l'Alisier dans diverses situations stationnelles}

\section{- Milieux à substrat calcaire}

Dans le quart Nord-Est de la France (Lorraine, Champagne, Bourgogne, Franche-Comté, Alsace), ces milieux portent actuellement le plus fort potentiel de production d'Alisier torminal, quantitativement et qualitativement. Des facteurs sylvicoles historiques y sont pour beaucoup:

- importance du taillis-sous-futaie, qui persiste encore de nos jours (surtout en forêts communales) ;

— potentialités pour l'Alisier globalement bonnes à excellentes, et s'exprimant “ spontanément » assez bien ;

- en conséquence, formation d'une tradition de conservation de l'Alisier (ne serait-ce parfois que pour la chasse), dont les effets sont encore visibles.

De bonnes potentialités ont également été constatées en Ile-de-France, Normandie, PoitouCharentes, Midi-Pyrénées, Provence-Alpes.

Le principal facteur limitant des potentialites de l'Alisier torminal sur substrat calcaire est l'alimentation en eau, avec une décroissance régulière des performances vers les types stationnels secs (des sols bruns eutrophes aux rendzines). Le Cormier et l'Alisier blanc fournissent alors un très intéressant complément du Torminal. 
Un cas très particulier est présenté par les sols bruns carbonatés ou calciques sur lœess récent ; les potentialités y sont excellentes, mais presque totalement masquees par la concurrence (c'est dans ces conditions que s'est développé un sujet de $32 \mathrm{~m}$ dans le Sundgau, le plus grand connu).

\section{- Milieux à substrat marneux et sols hydromorphes}

Cette situation se présente principalement en Woëvre, en Champagne humide et surtout sur le Plateau lorrain, où elle a fait l'objet des études de Girard (voir plus loin l'article de Le Goff et al., p. 243 et de Wilhelm, p. 364).

Les types de sols rencontrés sont des pélosols et variantes (sur marne pure ou avec légère incorporation de limon en surface), et des pélosols-pseudogleys [situation à faible couverture limoneuse $(<30 \mathrm{~cm})$ et discontinuité texturale brutale]. Dans les deux cas (surtout le second), il existe une contrainte liée au régime hydrique contrasté: engorgement hivernal, sécheresse estivale (aggravée par l'enracinement superficiel) ; les pélosols sans limon sont en outre des sols très lourds (teneur en argile supérieure à $45 \%$ ).

Les potentialités de l'Alisier torminal dans ces conditions sont bonnes à très bonnes, surtout par rapport aux autres feuillus. Les croissances varient beaucoup: excellentes pour les pélosols, les plus mauvaises pour les pélosols-pseudogleys. Dans tous les cas, on trouve des arbres de belle taille et bien conformés. Dans le Nord-Est, c'est une des rares situations écologiques où le Hêtre ne tende pas trop à exercer son hégémonie; grâce à cette concurrence réduite, la présence de l'Alisier est en général déjà importante.

- Milieux à sols acides ou lessivés

Situation surtout rencontrée dans le Centre, en Normandie, lle-de-France, Poitou-Charentes.

Les potentialités de l'Alisier y sont très variables, souvent difficiles à apprécier pour plusieurs raisons :

- complexité des interactions de facteurs stationnels: hydromorphie, sécheresse, oligotrophie, particularités du matériau (texture argileuse à très grossière, structure souvent dégradée, présence d'horizons indurés ou de lits de cailloux);

- extrême variété des structures de peuplements: de la haute futaie régulière à des formations très dégradées, basses, ouvertes - avec tout ce que cela implique de variations du niveau de concurrence des houppiers.

C'est dans les milieux à sols acides que la différence de dynamisme entre l'Alisier torminal et d'autres essences (Chêne sessile, Hêtre) est la plus forte, et qu'il peut être le plus réprimé par la concurrence - de sorte qu'il s'exprime comparativement mieux dans certaines conditions stationnelles particulièrement contraignantes, où les peuplements restent bas et peu denses. Corollairement, l'Alisier a eu par le passé des traitements très variés: mise en réserve, parfois (beaucoup plus rarement que sur les calcaires ou marnes du Nord-Est) élimination, maintien délibéré en sous-étage; le plus souvent, absence totale d'égards.

En conséquence, c'est dans ces milieux qu'il serait le plus souhaitable d'approfondir les recherches et de procéder à des expérimentations sylviculturales. Car l'Alisier manifeste d'ores et déjà d'excellentes potentialités dans certains cas, et il peut encore être précieux (bien qu'ayant un nettement moins bon potentiel de croissance) dans des mileux très contraignants où il n'y a guère d'alternative économiquement intéressante.

Les substrats rencontrés vont de matèriaux sédimentaires très variés, souvent grossiers (Centre), à divers produits de décarbonatation acidifiés (argile à silex, "limons des plateaux").

Types de sols:

- sols bruns acides, sols bruns lessivés légèrement acidifiés : la concurrence est forte sur ces sols non dégradés, globalement favorables à la forêt ; 
- sols lessivés acides plus ou moins dégradés (voire podzolisés) et hydromorphes, à régime hydrique souvent très contrasté.

Les humus vont du mull oligotrophe au moder (voire dysmoder).

Les performances de l'Alisier torminal décroissent avec le niveau de nutrition minérale; mais de très beaux arbres existent jusque sur sol lessivé à moder, surtout si l'alimentation hydrique est convenable. Une fertilisation serait en tout cas très profitable à l'Alisier dans ces conditions stationnelles (modalités à préciser, mais au moins pour les jeunes plants).

Dans l'Est de la France (sur substrat gréseux en particulier), l'Alisier torminal est quasiment absent des milieux acides ; comme dans le Nord du pays (voir $\S$ Froid, p. 238), c'est sans doute moins l'effet d'une incompatibilité fondamentale que d'une concurrence exacerbée par :

- la combinaison de la contrainte trophique à un climat moins favorable à l'Alisier qu'à d'autres essences ;

- l'importance de la conversion en futaie régulière;

- la domination habituelle de ces milieux par le Hêtre; celle du Chêne sessile, moins défavorable à l'Alisier, est plutôt réservée à des conditions stationnelles très contraignantes, ou bien résulte d'une intensité de sylviculture qui n'aura pu profiter à l'Alisier.

\section{- Milieux très fertiles}

Leur correspondent tous types de sols profonds, à bonnes conditions de nutrition minérale et surtout hydrique. En particulier:

- sols à couverture décarbonatée ou placages limoneux épais et non dégradés (éventuellement déjà légèrement acidifiés), sur matériaux divers : calcaire, marnes...;

- sols alluviaux ou colluviaux profonds et sains.

Dans ces conditions, I'Alisier torminal est rare ou absent, alors qu'il peut être abondant à une faible distance (on peut particulièrement le constater dans le cas de placages limoneux s'interrompant brutalement); et quand il est présent, l'Alisier est souvent chétif.

Mais ce ne sont encore qu'effets de la concurrence. Parfois, des sujets exceptionnels et "chanceux" sont là pour le prouver, donnant la pleine mesure des potentialités de l'essence.

- Milieux à sols hydromorphes

L'Alisier torminal a montré de très bonnes potentialitès sur des sols à engorgement même très superficiel, mais toujours temporaire (pélosols et pélosols-pseudogleys, sols lessivés à pseudogley). Les propriétés fonctionnelles des sols hydromorphes étant impossibles ou très difficiles à apprécier sans un suivi de leurs nappes, il serait encore très opportun de faire des études approfondies sur le comportement de l'Alisier dans ce type de milieux (dont le problème rejoint souvent celui des milieux acides).

L'Alisier torminal a vraisemblablement des potentialités interessantes sur des sols à engorgement permanent et profond (sols alluviaux); mais ceci reste à expérimenter, faute apparemment de cas à observer.

\section{CONCLUSIONS}

L'étude de l'Alisier torminal fournit un excellent exemple de l'importance que peuvent prendre les aspects synécologiques dans l'écologie d'une espèce végétale. Dans le cas présent, ne pas 
en tenir compte aurait conduit à négliger une part importante des potentialités d'une essence forestière de grande valeur.

Plus généralement, ce constat doit faire utiliser avec circonspection la notion d'affinités écologiques et de valeur indicatrice d'une espèce, tant pour sa détermination dans une région que pour son extrapolation en dehors des limites de celle-ci.

N. DRAPIER
Etudiant en thèse
(Université de Nancy 1)
Laboratoire de Recherches en Sciences forestières
ÉCOLE NATIONALE DU GÉNIE RURAL,
DES EAUX ET DES FORETS
14, rue Girardet
54042 NANCY CEDEX

\section{BIBLIOGRAPHIE}

Collectif. - Stations forestières, production et qualité des bois : éléments méthodologiques. - Nogent-surVernisson: CEMAGREF - Groupe de travail sur la typologie des stations forestières, 1989. $-254 \mathrm{p}$.

CRAVE (M.F.). - Un fruitier méconnu: l'Alisier torminal. - Forêt-Entreprise, n 28, 1985, pp. 14-20.

DRAPIER (N.), SEVRIN (E.). - Rapport d'études sur l'Alisier torminal. Données générales sur l'écologie, qualités du bois, applications à la sylviculture et à la prise en compte d'espéces voisines. - Nancy: École nationale du Génie rural, des Eaux et des Forêts - Laboratoire de Recherches en Sciences forestiéres, 1991. - 47 p. + annexes.

GIRARD (S.). - Comportement de l'Alisier torminal sur des sols à engorgement temporaire. Comparaison avec les Chênes pédonculé et sessile. - Champenoux: INRA - Centre de recherches de Nancy; Nancy: Université de Nancy I, 1991 . - 50 p. (rapport de stage de DEA de Biologie végétale et forestière).

GUINIER (Ph.) et al. - Technique forestière. - Paris : La Maison rustique, 1947. - 316 p. (3 édition, 1963 ).

KAUSCH-BLECKEN VON SCHMELING (W.). - Zwei Beiträge zur Elsbeere (Sorbus torminalis L. Crantz). 1 - Verbreitung der Elsbeere in Plessforst (Kreis Göttingen). 2 - Darstellung der Elsbeere in der Literatur von der Antike bis zum 18. Jahrhundert. - Plesse Archiv. Heft 17, 1981, pp. 95-160.

KAUSCH-BLECKEN VON SCHMELING (W.), MEYER (U.). - Die Elsbeere. - Aus dem Walde, Heft 33, 1980, $199 \mathrm{p}$.

LACHAUD (S.), MANSOURI (A.). - Activité cambiale, xylagènèse et structure du bois chez Sorbus torminalis L. Crantz. - Poitiers : Université de Poitiers - Laboratoire de Physiologie et Biochimie végétales, 1991. -
$32 \mathrm{p}$.

LANIER (L.), RAMEAU (J.-C.), KELLER (R.), JOLY (H.I.), DRAPIER (N.), SEVRIN (E.). - L'Alisier torminal Sorbus torminalis (L.) Crantz. - Revue forestière française, vol. XLII, $\mathrm{n}^{\circ} 1,1990$, pp. 13-34.

LORENTZ (M.), PARADE (A.). - Cours élémentaire de culture des bois. $4^{\mathrm{e}}$ édition revue et augmentée. Paris: Bouchard-Hyard; Nancy: Grimblot, Veuve Raybois et Cie, 1860. - $701 \mathrm{p}$.

LOUNI (D.). - Croissance de l'Alisier torminal Sorbus torminalis (L.) Crantz. - Nancy : Université de Nancy I Laboratoire de Biologie des Ligneux, 1990. - 23 p.

MATHIEU (A.). - Flore forestière. $4^{e}$ édition revue par P. Fliche. - Paris : J.-B. Baillière et Fils ; Nancy : Jacques, 1897. - XXXII + $705 \mathrm{p}$.

MAURANGES (P.). - L'Alisier torminal (Sorbus torminalis (L.) Crantz). - Nancy: École nationale du Génie rural, des Eaux et des Forêts, 1981. - 38 p. + annexes (Thème personnel).

NICLOUX (C.). - Potentialités des stations forestières des plateaux calcaires de Lorraine et des marnes du Keuper du Plateau lorrain pour l'Alisier torminal. Notes sur le Sorbier domestique. — Metz : Centre régional de la Propriété forestière, 1988. - V-61 p. (Raoport de stage de $2^{\Theta}$ année ENGREF).

PLEINES (V.). - Observation du domaine d'apparition écologique de l'Alisier torminal, de son comportement dans la végétation, et appréciation de son importance sylvicole. - Zürich : École polytechnique fédérale de Zürich, Institut de Recherches de la Forêt et du Bois, 1982. - 118 p. + annexes (travail de diplôme en sylviculture).

RAMEAU (J.-C.). - Le Tapis végétal. - Nancy : Ėcole nationale du Génie rural, des Eaux et des Forêts, 1988. -101 p. + annexes.

ROISIN (P.). - Le Domaine phytogéographique atlantique d'Europe. - Gembloux: Duculot, $1969 .-262$ p.

SAUVÉ (A.). - L'Alisier torminal en Poitou-Charentes. - Forêt-Entreprise, n² 28, 1985, pp. $20-22$.

SEVRIN (E.). - L'Alisier torminal (Sorbus torminalis (L.) Crantz) : qualité du bois et conditions de croissance. - Nancy: École nationale du Génie rural, des Eaux et des Forêts, 1992. - 250 p. + annexes (Thèse ENGREF en Sciences forestières). 